\title{
Cool Stars in the Hertzsprung-Russell Diagram
}

\author{
Jacco Th. van Loon \\ Lennard-Jones Laboratories, Keele University, ST5 5BG, UK \\ email: j.t.van.loon@keele.ac.uk
}

\begin{abstract}
As the opening review to the focus meeting "Stellar Behemoths: Red Supergiants across the Local Universe", I here provide a brief introduction to red supergiants, setting the stage for subsequent contributions. I highlight some recent activity in the field, and identify areas of progress, areas where progress is needed, and how such progress might be achieved.
\end{abstract}

Keywords. binaries: close, stars: evolution, Hertzsprung-Russell diagram, stars: late-type, stars: mass loss, stars: rotation, supergiants, supernovae: general, stars: winds, outflows

\section{Introduction}

Rooted in the book of Job, "Behemoth" is a worthy accolade to bestow upon the class of red supergiants. This finds reason in the popular use of the word. Thinking of the namesake Polish death metal band, red supergiants are dying stars, and their explosive death sparks the production and distribution of metals. In the cult game Final Fantasy, behemoths are beasts most feared for their magic spell "meteor"; indeed, red supergiants fabricate dust grains, a process enshrouded in mystery. Princeton University's Farlex WordNet describes behemoths as "abnormally large and powerful", "unusual", "of exceptional importance and reputation", and that "strongly influence the course of events" - all applicable to red supergiants. And they are, simply put, "whoppers".

Descendent from stars with birth masses in the region of $8-30 \mathrm{M}_{\odot}$, red supergiants become large, hundreds of $\mathrm{R}_{\odot}$ up to more than a thousand $\mathrm{R}_{\odot}$, because their mantles become convective. The reason they become convective is because the energy production rate in the core can no longer be balanced by radiative diffusion and instead bulk motion is induced to transport the energy to the surface. This causes a drastic restructuring of the mantle, with a much shallower density gradient. It becomes optically thin at much larger radial distance from the core, and hence the temperature of the plasma where this happens is relatively low, $T_{\text {eff }} \sim 3500-4000 \mathrm{~K}$, such that equilibrium is maintained $\left(L_{\text {emitted }}=L_{\text {produced }}\right)$.

To put this in perspective, a massive main sequence star would easily fit within the orbit of Mercury, but its red supergiant progeny could swallow Jupiter. At that point, it would take more than a decade to travel around its surface at a speed of $10 \mathrm{~km} \mathrm{~s}$, which is little more than the thermal motions in the star's atmosphere but only a few times smaller than the escape speed from its surface. Hence red supergiants oscillate on timescales of years, and their winds have speeds of just a few tens of $\mathrm{km} \mathrm{s}^{-1}$ taking tens of thousands of years for matter to move out to one parsec distance. This means that we may be able to read the immediate past of the red supergiant - even if it is no longer there - in the story told by its circumstellar medium. The low escape speed makes it easy for these stars to lose mass, but it critically depends on how long they spend their time doing this, how much mass they eventually will have lost. It also means that rotation at the mere $\mathrm{km} \mathrm{s}^{-1}$ level may already affect these stars. 
Red supergiants matter, as actors and probes in processes that drive galaxy evolution. But there are some important aspects of their formation, evolution and behaviour that need to be better understood:

\begin{tabular}{ll}
\hline importance of red supergiants & related issues to be resolved \\
\hline they return gas (and form dust) & $\rightarrow$ how much? \\
they lock up baryonic matter in remnants & $\rightarrow$ how much? \\
they are supernova progenitors & $\rightarrow$ which ones cause what type? \\
they are signposts of young populations & $\rightarrow$ what are their birth masses and lifetimes? \\
they stand out at infrared wavelengths & $\rightarrow$ how cool and bright do they really become? \\
they exhibit physics we do not understand & $\rightarrow$ convection, mass loss, binarity, ... \\
\hline
\end{tabular}

\section{Recent activity and progress in the field}

Stellar evolutionary models are becoming increasingly sophisticated. Yet none of them include all physics, e.g. either rotation or magnetic fields, and they often adopt different prescriptions for mixing (in particular the degree of "overshoot"). Even seemingly similar models can give rather different results, especially below $10000 \mathrm{~K}$ - the realm of the yellow hypergiants and red supergiants (Martins \& Palacios 2013). There is no agreement on the boundaries or overlap with the lower-mass stars on the asymptotic giant branch (AGB), exacerbated by the uncertainty regarding the super-AGB stars which are in many ways akin to AGB stars but which do continue to burn carbon in their core, and of which the ultimate fate - electron-capture supernova or white dwarf - remains elusive.

One way in which the model results differ is in the occurrence and extent of the blue loops, red supergiants becoming blue supergiant again before either exploding or becoming red supergiant for a second time. Of course, SN 1987A is exactly such case, having surprised everyone by being blue at the time of explosion yet displaying a ring of material as a wink to its red supergiant past. Dust was produced in it (Matsuura et al. 2011; Indebetouw et al. 2014) but, ultimately, more dust - be it produced in the supernova ejecta, red supergiant or present in the nearby interstellar medium (ISM) will have been destroyed by the supernova remnant (Lakićević et al. 2015; Temim et al. 2015). Theory indicates that rotation can encourage the migration of red supergiants to the blue part in the Hertzsprung-Russell diagram (Saio, Georgy \& Meynet 2013).

SN 1987A may once have looked much like the nearby red supergiant Betelgeuse (van Loon 2013). It is so close that we can see the convection cells bubbling up at its surface (Haubois et al. 2009), the clouds form in its extended atmosphere (Kervella et al. 2009, 2011), and the effects of it - and of its progenitor - on the local ISM (Decin et al. 2012).

Red supergiants lose mass at rates of $10^{-6}$ to $10^{-4} \mathrm{M}_{\odot} \mathrm{yr}^{-1}$, similar to - or in excess of - the nuclear burning rates (van Loon et al. 1999, 2005). This means that the exact rate of mass loss will determine the lifetime of the red supergiant phase as well as the total mass that will have been lost (Meynet et al. 2015). Mass loss may happen mostly in intense, brief phases either at the very end of red supergiant evolution, or before or after in the eruptions of (not so) luminous blue variables (Rest et al. 2012). Clearly, previous mass loss will affect the red supergiant phase, and mass loss during the red supergiant phase will affect subsequent evolution and the characteristics of the ensuing supernova as it proceeds through the circumburst environment (van Loon 2010).

One of the most important breakthroughs in stellar evolution observations has been the identification of the progenitors of supernovæ. This has been limited almost exclusively to the common type II plateau variety, confirming that they arise from red supergiants 
with birth masses in the range $8-17 \mathrm{M}_{\odot}$ (Smartt 2015). What happens to more massive red supergiants remains a mystery.

Studies of extra-galactic populations of red supergiants have enabled progress in our understanding of stellar evolution (Levesque et al. 2006, 2007) and stellar mass loss, and in reconstructing the star formation history and mapping the stellar feedback in spiral galaxies (Javadi et al. 2015). This will benefit from facilities such as JWST and E-ELT.

\section{Concluding remarks - how to move forward?}

Given the above, several areas of high priority emerge, defining possible strategies for progress. These are not limited to the following short list that I would like to advocate:

1 - theoretical studies of mass loss mechanisms;

2 - surveys of the red-to-blue supergiant ratio, including pulsation characteristics and surface abundances;

3 - (continued) surveys for supernova progenitors;

4 - determine the red supergiant luminosity functions in galaxies with differing metal content and recent (spatially varying) star formation histories;

5 - high resolution, high precision case studies in combination with three-dimensional computational models.

And finally, if close binary interaction does affect the evolution of a large fraction of the massive star population (de Mink et al. (2014), then observational and theoretical efforts must be promoted that aim to quantify these effects.

\section{References}

Decin, L., et al. 2012, A\&A, 548, 113

de Mink, S. E., Sana, H., Langer, N., Izzard, R. G., \& Schneider, F. R. N. 2014, ApJ, 782, 7

Haubois, X., et al. 2009, A\&GA, 508, 923

Indebetouw, R., et al. 2014, ApJ, 782, L2

Javadi, A., Saberi, M., van Loon, J. T.h., Khosroshahi, H., Golabatooni, N., \& Mirtorabi, M. T. 2015, MNRAS, 447, 3973

Kervella, P., Verhoelst, T., Ridgway, S. T., Perrin, G., Lacour, S., Cami, J., \& Haubois, X. 2009, $A \mathscr{G} A, 504,115$

Kervella, P., Perrin, G., Chiavassa, A., Ridgway, S. T., Cami, J., Haubois, X., \& Verhoelst, T. 2011, A\&A, 531, 117

Lakićević, M., et al. 2015, ApJ, 799, 50

Levesque, E. M., Massey, P., Olsen, K. A. G., Plez, B., Meynet, G., \& Maeder, A. 2006, ApJ, 645,1102

Levesque, E. M., Massey, P., Olsen, K. A. G., \& Plez, B. 2007, ApJ, 667, 202

Martins, F. \& Palacios, A. 2013, A\& A, 560, 16

Matsuura, A., et al. 2011, Science, 333, 1258

Meynet, G., et al. 2015, A\&A, 575, 60

Rest, A., et al. 2012, Nature, 482, 375

Saio, H., Georgy, C., \& Meynet, G. 2013, MNRAS, 433, 1246

Smartt, S. J. 2015, PASA, 32, 16

Temim, T., Slane, P., Tchernyshyov, K., Boyer, M. L., Meixner, M., Gall, C., \& Roman-Duval, J. 2015, ApJ, 799, 158

van Loon, J. T.h. 2010, $A S P C, 425,279$

van Loon, J. T.h. 2013, EAS, 60, 307

van Loon, J. T.h., et al. 1999, A\&SA, 351, 559

van Loon, J. T.h., Cioni, M.-R. L., Zijlstra, A. A., \& Loup, C. 2005, A\& A, 438, 273 